\title{
Sliding Mode Thermal Control System for Space Station Furnace Facility
}

\author{
Mark E. Jackson, Member, IEEE and Yuri B. Shtessel, Member, IEEE
}

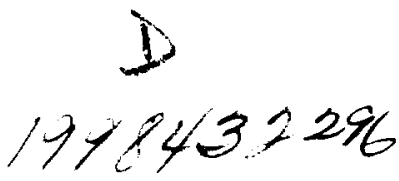

\begin{abstract}
This paper addresses the decoupled control of the nonlinear, multiinput-multioutput, and highly coupled space station furnace facility (SSFF) thermal control system. Sliding mode control theory, a subset of variable-structure control theory, is employed to increase the performance, robustness, and reliability of the SSFF's currently designed control system. This paper presents the nonlinear thermal control system description and develops the sliding mode controllers that cause the interconnectedw subsystems to operate in their local sliding modes, resulting in control system invariance to plant uncertainties and external and interaction disturbances. The desired decoupled flow-rate tracking is achieved by optimization of the local linear sliding mode equations. The controllers are implemented digitally and extensive simulation results are presented to show the flowrate tracking robustness and invariance to plant uncertainties, nonlinearities, external disturbances, and variations of the system pressure supplied to the controlled subsystems.
\end{abstract}

Index Terms- Decoupled control, nonlinear control, robust control, sliding mode control, thermal control.

\section{INTRODUCTION}

$\mathbf{T}$ HE space station furnace facility (SSFF) provides the necessary core systems to operate various material processing furnaces in the microgravity environment of the International Space Station Alpha. The thermal control system (TCS) is defined as one of the core systems, and its function is to collect excess heat from furnaces and to provide precise cold temperature control of components and of certain furnace zones. The TCS utilizes single phase water as its cooling medium and consists of piping, heat exchangers, coldplates, cooling jackets, pressure, flow, and temperature sensors, and a centrifugal pump. The TCS schematic is illustrated in Fig. 1.

The objective of the TCS is to simultaneously control the thermal environments of several furnaces and related subsystems plumbed in parallel. Specifically, the high accuracy, robust, and decoupled tracking of time-varying flow-rate profiles in paths 1, 2, and 3 is desired. The flow-rate through path 4 is passively controlled to a constant by fixing its valve position and by maintaining the system pressure drop constant.

The current SSFF control system consists of four linear proportional integral derivative (PID) controllers [5]. Three of these track flow profiles in paths 1,2 and 3 , respectively,

Manuscript received July 18,1996 . Recommended by Associate Editor, T. Ogunnaike.

M. E. Jackson is with the National Aeronautics and Space Administration, Marshall Space Flight Center, Huntsville, AL 35807 USA.

Y. B. Shtessel is with the Department of Electrical and Computer Engineering, University of Alabama, Huntsville. AL 35807 USA.

Publisher Item Identifier S 1063-6536(98)06403-3. and one controls the system pressure by modulated the pump's speed based on sensed system pressure. System pressure control serves to decouple the flow dynamics between respective paths, which aids in flow tracking performance.

The SSFF will remain aboard the Space Station for at least 15 years. As a result of this long duration mission, the loss or degradation of pressure control is a likely event. This event would cripple flow tracking performance and would result in a total SSFF shut down. Reliability of such systems can be improved and performance maintained by the use of powerful nonlinear control methods. The most competitive methods are feedback linearization and sliding mode control. Both methods can provide desired linear performance to nonlinear systems; however, feedback linearization is more sensitive to uncertainties and disturbances and is more complicated to implement than sliding mode control. This article shows how sliding mode control will improve reliability as well as performance, without adding system complexity.

According to Itkis [1], Utkin [2], and DeCarlo [3], sliding mode control is unique from other linear and nonlinear control methods in its ability to achieve accurate, robust, decoupled tracking for a class of nonlinear time-varying multiinput-multioutput systems in the presence of disturbances and parameter variations.

Section II formulates the TCS problem including a description of the nonlinear multiinput-multioutput system mathematical model with partially known parameters. Section III develops the sliding mode control theory in the context of the TCS problem. Section IV discusses the sliding mode realization. In Section V, the simulation results are presented to demonstrate sliding mode's increased tracking performance, robustness, and reliability compared with the linear TCS controllers.

\section{TCS PROBLEM FORMULATION}

This section formulates the TCS control problem upon which the sliding mode controllers are designed to solve. The problem formulation begins with the derivation of the TCS mathematical model.

\section{A. TCS Mathematical Model}

The mathematical model of the TCS is derived as a means to simulate the dynamic response of the flows, pressures, and actuators within the system. The model is divided into hydraulic, actuator, and pump submodels, where each submodel parameter is defined in the Appendix. 


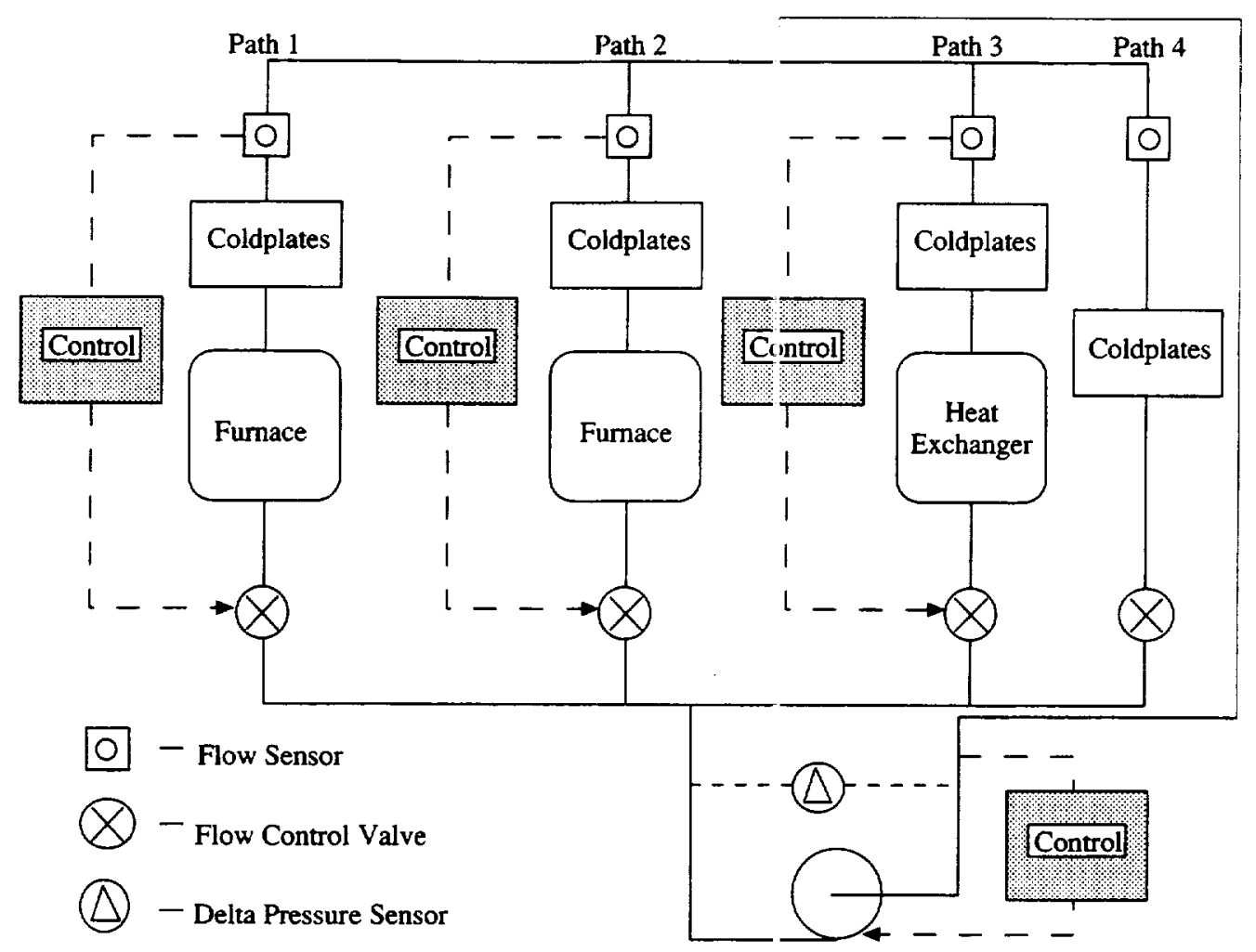

Fig. 1. TCS schematic.

1) Hydraulic Submodel: Hydraulic systems such as the TCS usually consists of liquid-filled tanks connected by pipes having orifices, valves, or other flow-restricting devices. The hydraulic mathematical model of the TCS is derived in terms of three basic elements: resistance, compliance (fluid capacitance), and inertance (fluid inertia) [4]. Shown in Fig. 2, these elements are combined to form an electrically equivalent circuit for the TCS model. From this circuit, the flow and pressure equations are written using Kirchoff's voltage law. The resulting equations are shown as

$$
\begin{aligned}
\dot{P} & =\frac{2}{C}\left(W_{S}-\sum_{i=1}^{4} W_{i}\right) \\
\dot{W}_{S} & =\frac{1}{L_{S}}\left(P_{S}-P-R_{S} W_{S}\right) \\
\dot{W}_{i} & =\frac{1}{L_{P_{i}}}\left[P-\left(R_{V_{i}}+R_{P_{i}}\right) W_{i}\right], \quad \forall i=\overline{1,4}
\end{aligned}
$$

where $P=P_{1}-P_{2}$ is the system pressure drop, $W_{S}$ is the pump source flow, $W_{i}$ are the path flow-rates, $R_{V_{i}}=\alpha e^{-\beta \theta_{i}}$ are the valve resistances, and $\theta_{i}$ are the angular valve positions.

2) Actuator Submodel: Each flow path has an actuator valve unit consisting of a ball valve, brushless $\mathrm{dc}$ motor, and built-in servo speed controller. The angular valve speed $\left(\omega_{i}\right)$ response of this unit to an input valve speed command $\left(u_{i}\right)$ is given by the actuator vendor [5] as a first-order lag. This is represented as

$$
\dot{\omega}_{i}=\frac{1}{\tau}\left(k_{v} u_{i}-\omega_{i}\right), \quad \forall i=\overline{1,4}
$$

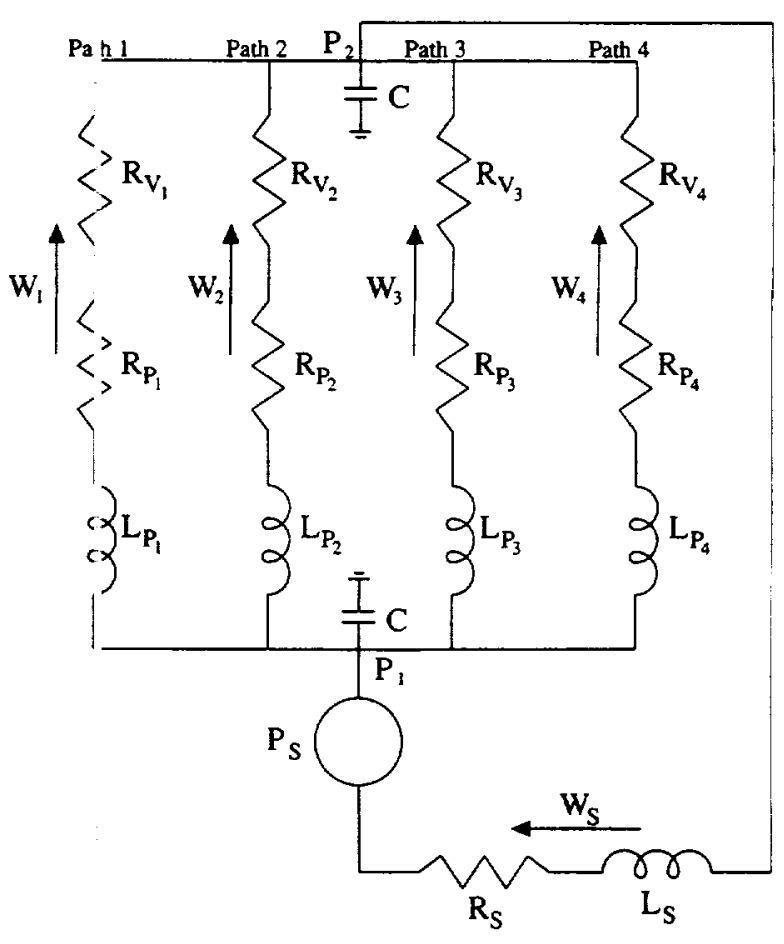

Fig. 2. TC i equivalent circuit.

The resulting valve speed is integrated to achieve the valve's position. 'This is modeled as

$$
\dot{\theta}_{i}=\omega_{i}, \quad \forall i=\overline{1,4} .
$$

The simple actuator model represented by (2) and (3) is an acceptable model for this problem because the actuator is not 

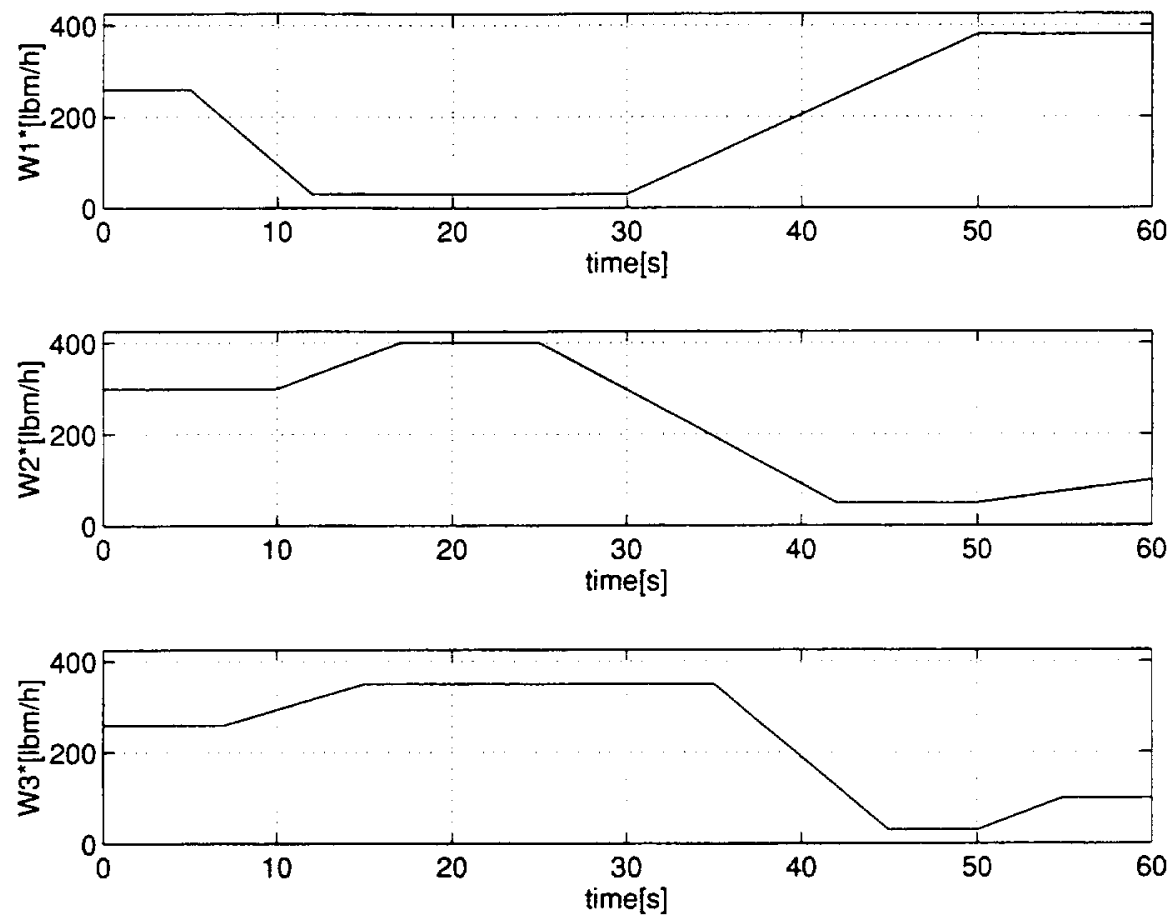

Fig. 3. TCS tracking profiles.

explicitly controlled, but only used as an effector whose gain and phase responses are adequately modeled.

3) Pump Submodel: The centrifugal pump model [5] consists of a pump motor, pump speed controller, and system pressure controller equations

$$
\begin{aligned}
\dot{\omega}_{p} & =\frac{1}{j_{m l}}\left(k_{t} I_{a}-k_{1} \omega_{p}^{2}\right) \\
\dot{I}_{a} & =\frac{1}{L_{M}}\left[-R_{m} I_{a}-k_{e} \omega_{p}-k_{2} P_{e}-k_{3} \omega_{f}+\boldsymbol{Q} \boldsymbol{X}_{c}\right] \\
\dot{\omega}_{f} & =\frac{1}{\tau_{f}}\left(\omega_{p}-\omega_{f}\right) \\
\dot{\boldsymbol{X}}_{c} & =\boldsymbol{M} \boldsymbol{X}_{c}+\boldsymbol{B} \cdot\left[\omega_{f} P P^{*}\right]^{T}
\end{aligned}
$$

where $\omega_{p}$ is the pump speed, $I_{a}$ is the pump motor armature current, $\omega_{f}$ is the pump speed feedback into the pump speed controller, $\boldsymbol{X}_{c}$ is the $2 \times 1$ vector of system pressure controller and pump speed controller states, and $P_{e}$ is the system pressure error $\left(P^{*}-P\right)$.

4) TCS Model Summary: The composite 19th-order multiinput-multioutput nonlinear TCS with parameter uncertainties, interaction disturbances (dynamic coupling between parallel paths), and external disturbances is represented by (1)-(4). The model contains three input valve commands $\left(u_{1}, u_{2}\right.$, and $\left.u_{3}\right)$ and three output flow-rates $\left(W_{1}, W_{2}\right.$, and $\left.W_{3}\right)$. The model is verified, through comparisons with test data from Space Station TCS tests, to adequately represent the salient dynamics of the SSFF TCS.

\section{B. Control System Objective}

The control system objective is to simultaneously control the thermal environments of several furnaces and related subsystems plumbed in parallel. Specifically, the high accuracy, robust, and decoupled tracking performance of time-varying flow-rate profiles $W_{i}^{*}(t)$ is desired. The problem is defined mathematically as

$$
\lim _{t \rightarrow \infty} e_{i}(t)=0, \quad \forall i=\overline{1,3}
$$

where

$$
e_{i}(t)=W_{i}^{*}(t)-W_{i}(t)
$$

The solution of this problem using sliding mode is two fold [6]. First, sliding surface equations

$$
\sigma_{i}(x)=0, \quad \forall i=\overline{1,3}
$$

are chosen such that the flow-rate tracking errors defined by (6) are the outputs of linear homogeneous systems with constant coefficients and with desired eigenvalues placements. Second, discontinuous control laws of the form

$$
u_{i}(t, x)=\left\{\begin{array}{ll}
u_{i}^{+}(t, x) & \sigma_{i}(x)>0 \\
u_{i}^{-}(t, x) & \sigma_{i}(x)>0
\end{array} \quad \forall i=\overline{1,3}\right.
$$

are chosen such that the desired output tracking motion goes to and stays on the sliding surfaces defined by (7).

\section{Sliding Mode Controller Design}

This section discusses the theory of sliding mode control as it is applied to the design of the TCS problem. This section develops the system transformation that is most useful in determining the system's vector relative degree and in analyzing the stability of the system's internal dynamics; synthesizes the sliding surfaces upon which the desired output responses are restricted; and develops the sliding mode controllers that force the system to move and to stay on the sliding surface for all subsequent time. 


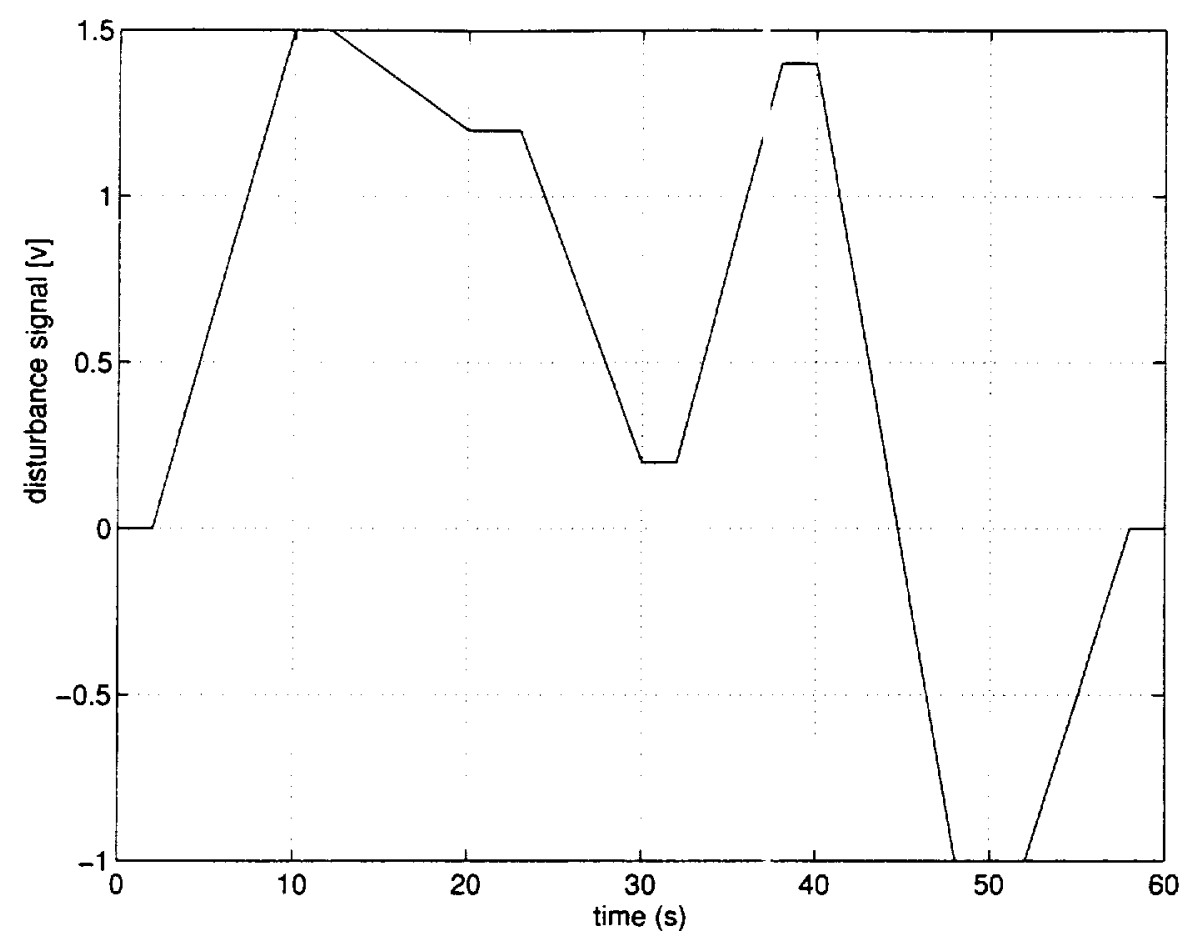

Fig. 4. Normalized external disturbance.

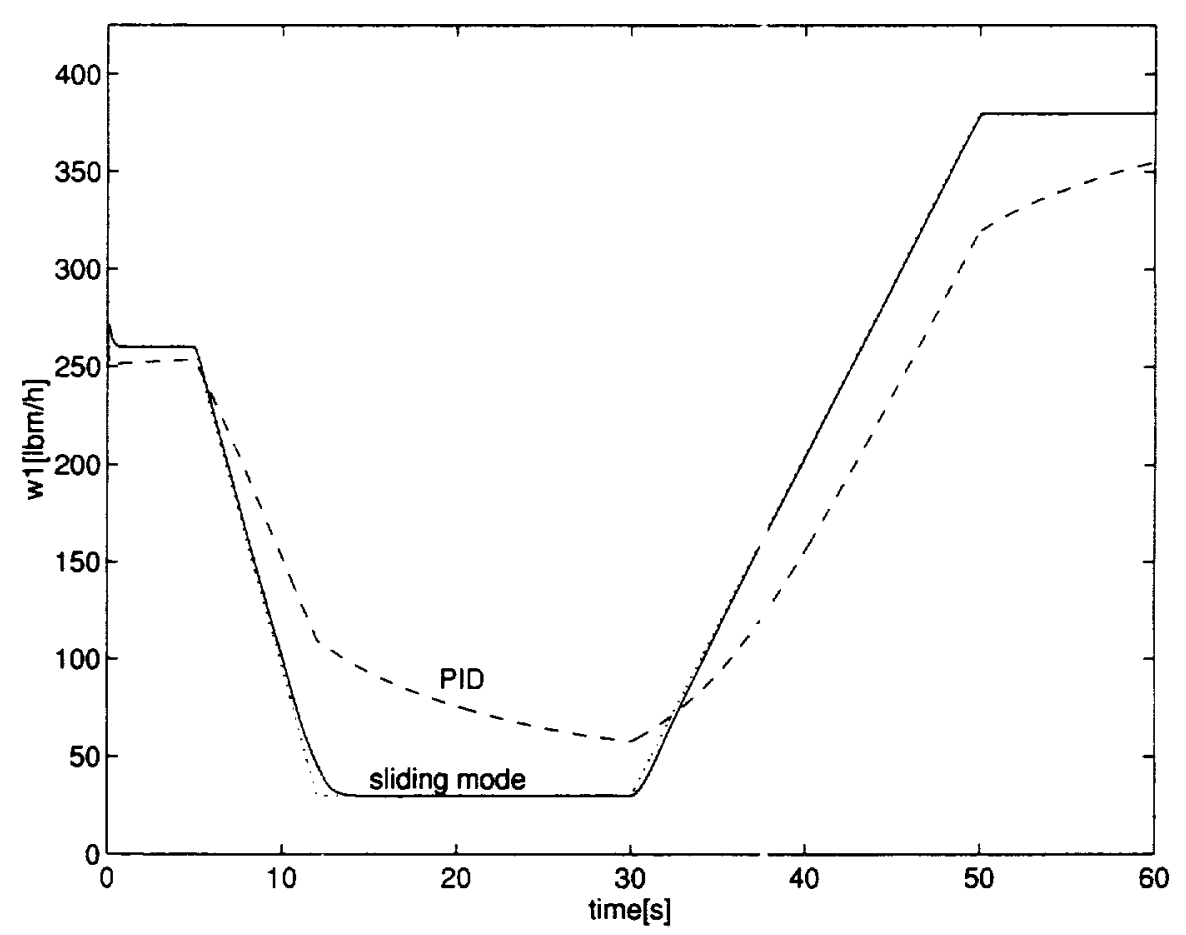

Fig. 5. Path 1 flow tracking response.

For the TCS output tracking problem, the linear homoge- where the relative degree $r_{i}$ for each output channel and the neous sliding surface equations are defined as

$$
\sigma_{i}(\boldsymbol{x})=\sum_{j=0}^{r_{i}-1} c_{j i} e_{i}^{(j)}, \quad \forall i=\overline{1,3}
$$
system's internal dynamics, if any, are determined through a system tra: ssformation. It is well known [7] that sliding mode control is applicable in systems with stable internal (zero or asymptotic zero) dynamics. 


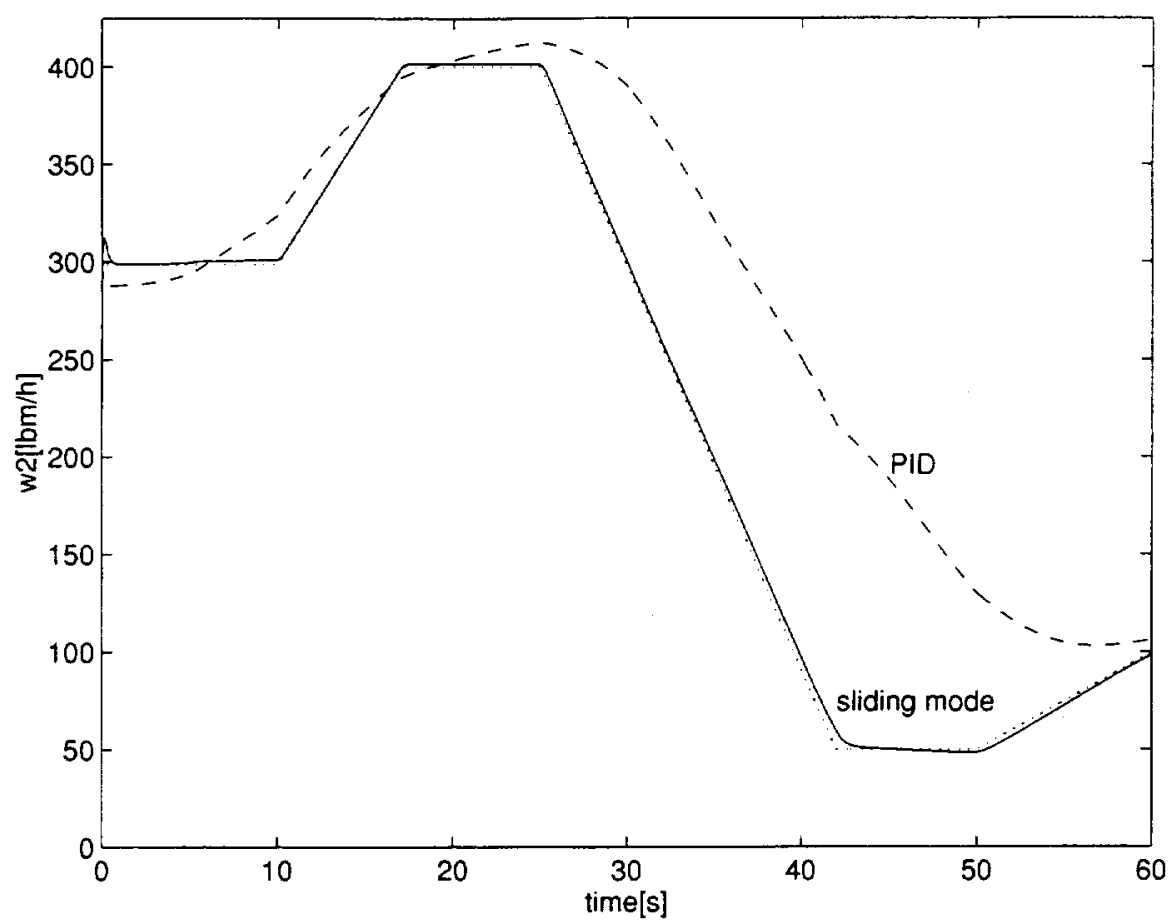

Fig. 6. Path 2 flow tracking response.

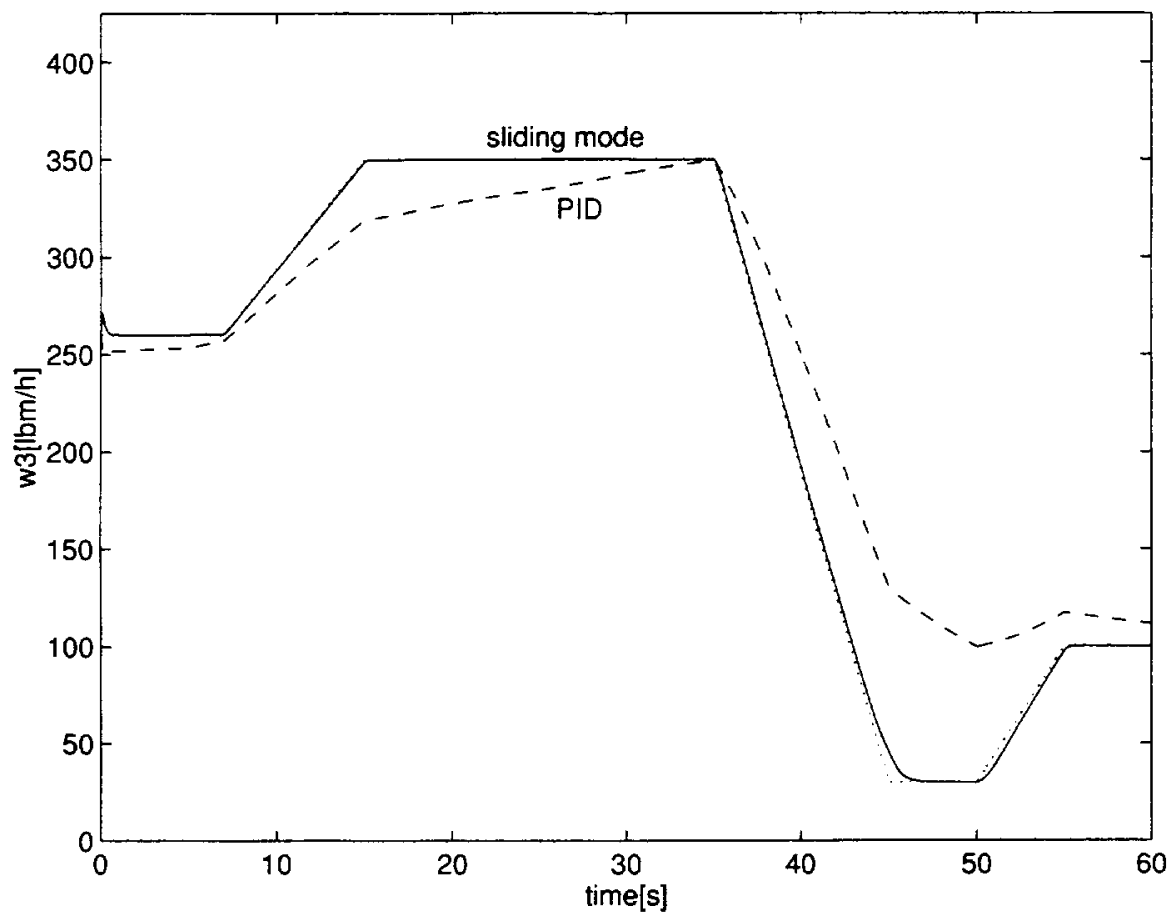

Fig. 7. Path 3 flow tracking response.

\section{A. System Transformation}

The 19th-order ( $n=19)$ TCS system represented by (1)-(4) is presented in matrix form

$$
\begin{aligned}
& \dot{\boldsymbol{x}}=\boldsymbol{f}(\boldsymbol{x})+\boldsymbol{G u} \\
& \boldsymbol{y}=\boldsymbol{h}(\boldsymbol{x})
\end{aligned}
$$

where $x$ is a $19 \times 1$ vector of model states, $f(x)$ is a smooth $19 \times 1$ vector field, $G$ is a constant $19 \times 3$ matrix, $u$ is a $3 \times$
1 vector of input commands, $y$ is a $3 \times 1$ vector of outputs, and $h(x)$ is a smooth $3 \times 1$ vector field.

The system transformation begins by deriving a direct input-to-output relationship. This is accomplished by taking successive derivatives of each output until at least one input appears [7]

$$
y_{i}^{\left(r_{i}\right)}=L_{f}^{r_{i}} h_{i}(\boldsymbol{x})+\sum_{j=1}^{m} L_{\boldsymbol{g}_{i}} L_{f}^{r_{i}-1} h_{i}(\boldsymbol{x}) u_{j}, \quad \forall i=\overline{1, m}
$$




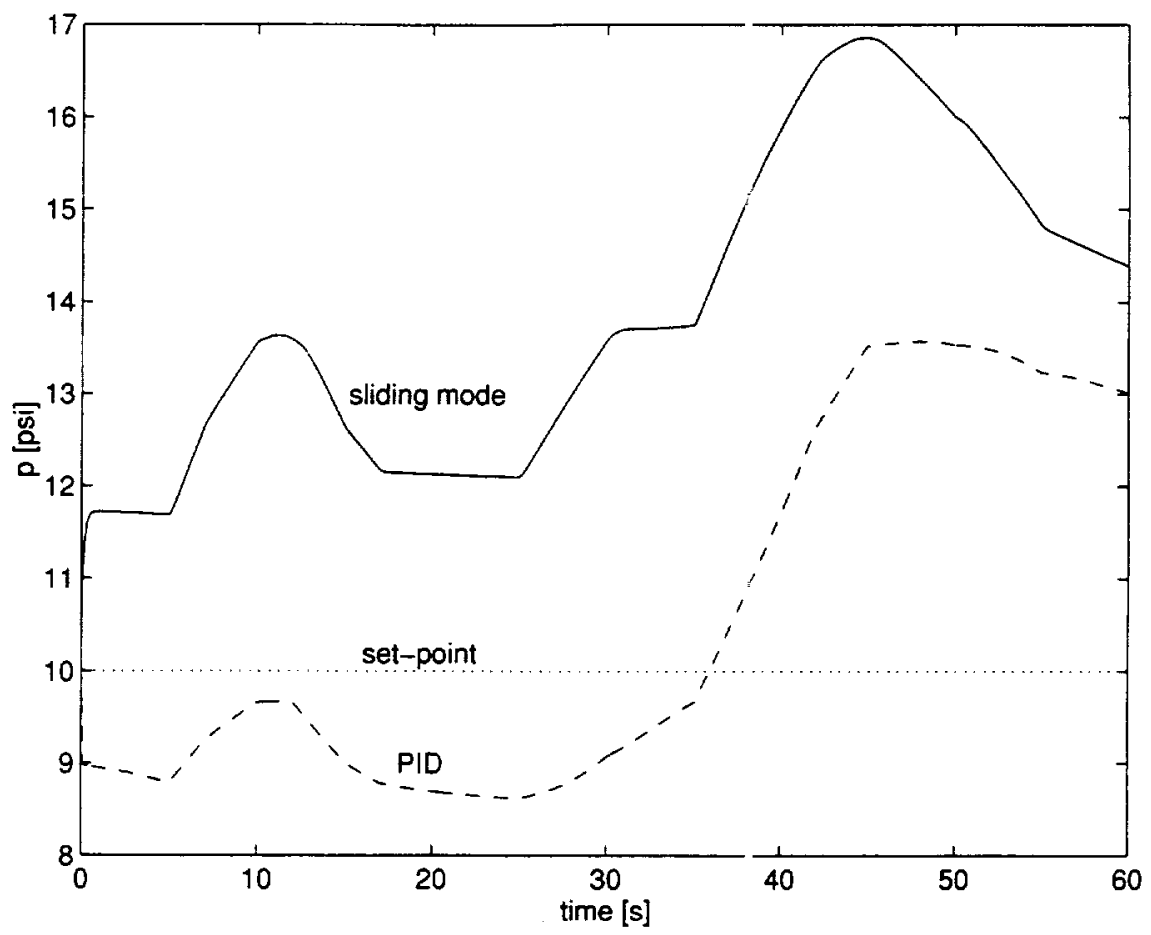

Fig. 8. System pressure response.

where the scalar function $L_{f} h$ is the Lie derivative [7] of $h$ with respect to $f, m$ is the number of inputs, $r_{i}$ is the number of differentiations required for the input the appear (called the "relative degree" of the $i$ th output channel), and $L_{g_{i}} L_{f}^{r_{i}-1} h_{i}(x) u_{j} \neq 0$ for at least one $j$. Applying (11) to (10) for $m=3$ yields the ninth-order $(r=9)$ input-output description

$$
\begin{aligned}
& {\left[\begin{array}{c}
\dddot{y}_{1} \\
\dddot{y}_{2} \\
\dddot{y}_{3}{ }_{3}
\end{array}\right]=} {\left[\begin{array}{l}
\sum_{j=1}^{4} \frac{\Psi_{j 1}}{L_{P_{1}}}+\frac{\alpha \beta W_{1}^{2} e^{-\beta \theta_{1}} \omega_{1}}{\tau L_{P_{1}}} \\
\sum_{j=1}^{4} \frac{\Psi_{j 2}}{L_{P_{2}}}+\frac{\alpha \beta W_{2}^{2} e^{-\beta \theta_{2}} \omega_{2}}{\tau L_{P_{2}}} \\
\sum_{j=1}^{4} \frac{\Psi_{j 3}}{L_{P_{3}}}+\frac{\alpha \beta W_{3}^{2} e^{-\beta \theta_{3}} \omega_{3}}{\tau L_{P_{3}}}
\end{array}\right] } \\
&+\boldsymbol{D}(\boldsymbol{x})\left[\begin{array}{l}
u_{1} \\
u_{2} \\
u_{3}
\end{array}\right]
\end{aligned}
$$

where we have (13), shown at the bottom of the page, and

$$
\Psi_{1 i}=-\alpha \beta^{2} \omega_{i}^{2} W_{i}^{2} e^{-\beta \theta_{i}}+\frac{2 \Omega_{1 i} W_{i}^{3}+2 \Omega_{1 i} W_{i}}{L_{P_{i}}} \alpha \beta \omega_{i} e^{-\beta \theta_{i}}
$$

$$
\begin{aligned}
\Psi_{2 i}= & \frac{2 \omega \beta \omega_{i} W_{i} \Omega_{1 i} e^{\beta \theta_{i}}}{L_{P_{i}}}+\frac{\Omega_{1 i}}{L_{P_{i}}^{2}}\left(4 W_{i}^{2} \Omega_{2 i}^{2}+2 \Omega_{1 i} \Omega_{2 i}\right) \\
& -\frac{2 \Omega_{1 i}}{L_{i} C} \\
\Psi_{3 i}= & \frac{4}{L_{P_{i}} C} \Omega_{2 i} W_{i}\left(W_{S}-\sum_{j=1}^{4} W_{j}\right) \\
\Psi_{4 i}= & =\frac{2}{C}\left[\frac{1}{L_{S}}\left(P_{S}-R_{S} W_{2}^{2}-P\right)-\sum_{\substack{k=1 \\
k \neq i}}^{4} \frac{\Omega_{1 i}}{L_{P_{k}}}\right] \\
\Omega_{1 i}= & =\Omega_{2 i} W_{i}^{2}+P \\
\Omega_{2 i}= & =-\left(\alpha e^{-\beta \theta_{i}}+K_{i}\right), \quad \forall i=\overline{1,3} .
\end{aligned}
$$

Matrix $I(x)$ is full rank and nonsingular for any operational mode of tie system. The system (12) has a vector relative degree of $-=[3,3,3]$ and a total relative degree of $r=9$. Since $r<\imath,(n-r)$ th or 10th-degree internal dynamics exist and shoulc be analyzed for stability.

The system (12) is then transformed to the phase-variable canonical form [8] and combined with the internal dynamics [dynamics not involved in (12)] to form the so-called "normal

$$
\boldsymbol{D}(\boldsymbol{x})=\left[\begin{array}{ccc}
\frac{\alpha \beta K_{v} W_{1}^{2}}{\tau L_{P_{1}}} e^{-\beta \theta_{1}} & 0 & 0 \\
0 & \frac{\alpha \beta K_{v} W_{2}^{2}}{\tau L_{P_{2}}} e^{-\beta \theta_{z}} & 0 \\
0 & 0 & \frac{\alpha \beta K_{v} W_{3}^{2}}{\tau L_{P_{3}}} e^{-\beta \theta_{3}}
\end{array}\right]
$$


form" representation of the original system

$$
\begin{aligned}
\dot{Z}_{1 i} & =Z_{2 i} \\
\dot{Z}_{2 i} & =Z_{3 i} \\
\dot{Z}_{3 i} & =\frac{1}{L_{P_{i}}}\left[\sum_{j=1}^{4} \Psi_{j i}+\frac{1}{\tau} \alpha \beta W_{i}^{2} e^{-\beta \theta_{i}}\left(\omega_{i}+K_{v} u_{i}\right)\right] \\
y_{i} & =Z_{1 i}, \quad \forall i=\overline{1,3} \\
\dot{\eta}_{k} & =q_{k}(Z, \eta), \quad \forall k=\overline{10,19}
\end{aligned}
$$

where $W_{i}, \theta_{i}$, and $\omega_{i}$ can be expressed through $Z_{1 i}, Z_{2 i}$, and $Z_{3 i}$ using (14)-(19). The internal dynamics $\eta$ consist of equations in (1)-(4) that represent the system pressure drop, pump source flow, pump, and path 4 submodels.

Stability of the internal (asymptotic zero) dynamics is shown using Lyapunov's first method [10]. This method involves linearizing the nonlinear internal dynamics throughout the operating range of the system. Eigenvalues at each operating point are calculating to assure that all have negative real parts [11].

\section{B. Sliding Surface Design}

The second major step in the design process is to choose the desired linear homogeneous sliding surfaces upon which the motion of the tracking errors (6) is restricted. According to (9), where $r_{i}=3, \forall i=\overline{1,3}$, the sliding surface equations are chosen as the set of second-order linear homogeneous differential equations with constant coefficients of the form

$$
\sigma_{i}(\boldsymbol{x})=\ddot{e}_{i}+c_{2 i} \dot{e}_{i}+c_{1 i} e_{i}=0, \quad \forall i=\overline{1,3} .
$$

The coefficients are chosen to obtain the desired transient response of the tracking error motion. These coefficients are chosen as $c_{1 i}=225.0$ and $c_{2 i}=21.0, \forall i=\overline{1,3}$, using the ITAE method [12]. According to the standard second-order form

$$
\ddot{e}+2 \zeta \omega_{n} \dot{e}+\omega_{n}^{2} e=0
$$

the sliding surface has a damping ratio of $\zeta=0.7$, a natural frequency of $\omega_{n}=15.0$, and a settling time of $4 / \zeta \omega_{n}=$ $0.38 \mathrm{~s}$. This represents a desired transient response of the system operating in sliding mode; however, there are several realization issues (for example, discrete sampling rate and unmodeled modes) that must be considered before this desired transient performance is obtained. Section IV will address these issues.

\section{Control Function Design}

The third major step is to design the control function to provide the existence of sliding mode on the sliding surfaces (21). The motion of the system in $\sigma$-space is described by the system

$$
\dot{\sigma}(\boldsymbol{x})=\frac{\partial \sigma(\boldsymbol{x})}{\partial \boldsymbol{x}} \dot{\boldsymbol{x}}=\frac{\partial \sigma(\boldsymbol{x})}{\partial \boldsymbol{x}}[\boldsymbol{f}(\boldsymbol{x})+\boldsymbol{G u}] .
$$

A candidate Lyapunov function [9] is introduced as

$$
V(x)=\frac{1}{2} \sigma^{T}(x) \sigma(x)
$$

where $V(\boldsymbol{x})$ is positive definite and

$$
\lim _{\|\sigma(\boldsymbol{x})\| \rightarrow \infty} V(\boldsymbol{x})=\infty .
$$

Global asymptotic stability of $\sigma(x)=0$ is assured if the derivative of (24) is negative definite [9]:

$$
\dot{V}(\boldsymbol{x})=\sigma^{T}(\boldsymbol{x}) \dot{\sigma}(\boldsymbol{x})<0 .
$$

Moreover, if the inequalities

$$
\sigma_{i}(\boldsymbol{x}) \dot{\sigma}_{i}(\boldsymbol{x})<-\rho_{i}\left|\sigma_{i}(\boldsymbol{x})\right|, \quad \rho_{i}>0, \quad \forall i=\overline{1,3}
$$

are satisfied, then finite sliding surface reaching times are calculated as

$$
t_{r i}=\frac{\left|\sigma_{i}(0)\right|}{\rho_{i}}, \quad \forall i=\overline{1,3} .
$$

Analysis of the system (23) shows that the matrix $[\partial \sigma(x) / \partial \boldsymbol{x}] \boldsymbol{G}$ is diagonal. This allows system (23) to be rewritten in decoupled form

$$
\dot{\sigma}_{i}(\boldsymbol{x})=\tilde{a}_{i}(\boldsymbol{x})-\tilde{b}_{i}(\boldsymbol{x}) u_{i}, \quad \forall i=\overline{1,3}
$$

where

$$
\begin{aligned}
\tilde{a}_{i}(\boldsymbol{x})= & \dddot{y}_{i}^{*}-\frac{1}{L_{P_{i}}}\left(\sum_{j=1}^{4} \Psi_{j i}+\frac{\alpha \beta \omega_{i} W_{i}^{2} e^{-\beta \theta_{i}}}{\tau}\right) \\
& +c_{2 i} \ddot{e}_{i}+c_{1 i} \dot{e} \\
\tilde{b}_{i}(\boldsymbol{x})= & \frac{\alpha \beta k_{v} W_{i}^{2} e^{-\beta \theta_{i}}}{L_{P_{i}} \tau}
\end{aligned}
$$

and $\tilde{b}_{i}(\boldsymbol{x})>0, \forall \boldsymbol{x}$. The discontinuous control law

$$
u_{i}(\boldsymbol{x})=\hat{u}_{i} \operatorname{sign}\left[\sigma_{i}(\boldsymbol{x})\right], \quad \forall i=\overline{1,3}
$$

where

$$
\begin{aligned}
\hat{u}_{i} & \geq\left|u_{i}^{e q}(\boldsymbol{x})+\frac{\rho_{i}}{\tilde{b}_{i}(\boldsymbol{x})}\right| \\
u_{i}^{e q}(\boldsymbol{x}) & =\left[\tilde{b}_{i}(\boldsymbol{x})\right]^{-1} \tilde{a}_{i}(\boldsymbol{x})
\end{aligned}
$$

satisfies inequality (27), $\forall i=\overline{1,3}$. This means that the control law (32) provides the existence of sliding mode on the sliding surfaces (21) with finite reaching times (28).

The equivalent control (34) is calculated through a typical flight simulation, including disturbances, and shown to be mostly contained within a \pm 5 volt boundary. Considering this boundary and that the actuator is designed to accommodate input control signals between $\pm 5 \mathrm{~V}, \hat{u}_{i}$ is chosen as $5.0 \mathrm{~V}$, and the resulting discontinuous control function (32) is shown as.

$$
u_{i}(\boldsymbol{x})=5 \cdot \operatorname{sign}\left[\sigma_{i}(\boldsymbol{x})\right], \quad \forall i=\overline{1,3} .
$$




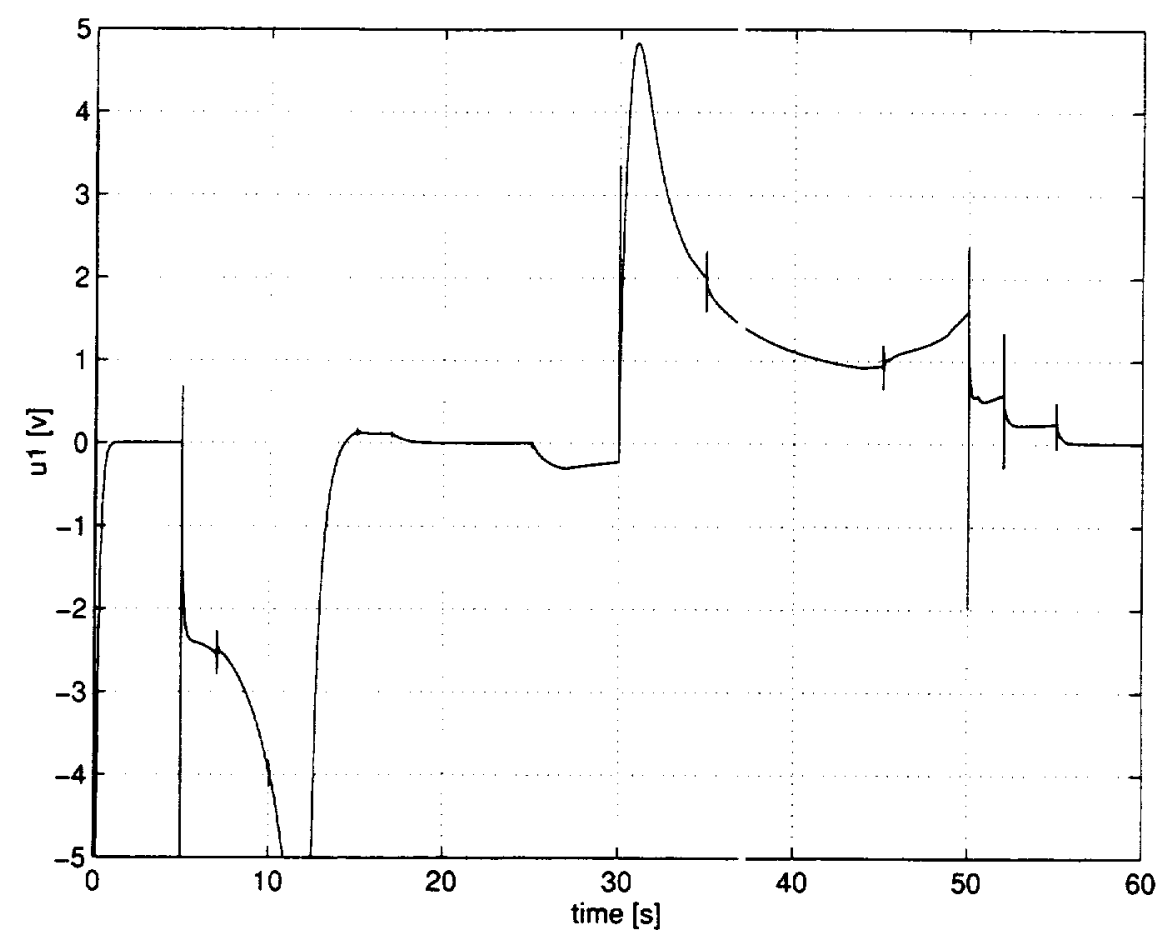

Fig. 9. Discrete sliding control input to path 1.

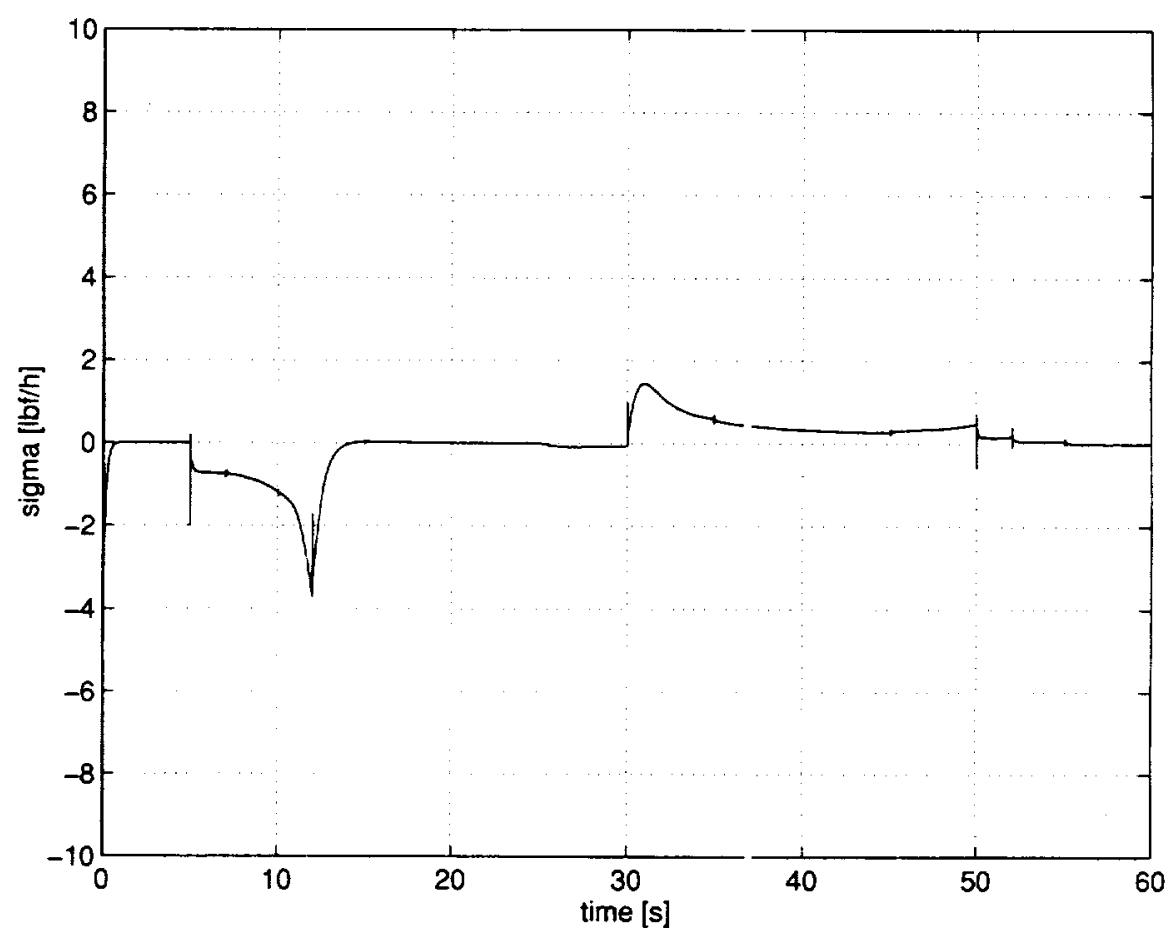

Fig. 10. Sliding surface response in path 1.

\section{Realization of SLiding Mode}

The SSFF computer samples measurements once every $T=0.005 \mathrm{~s}$ and utilizes 12-bit $\mathrm{A} / \mathrm{D}$ converters to digitize the data. It can be shown that the discrete sampling time $T=0.005 \mathrm{~s}$ satisfies the sufficient condition for the existence of sliding mode for systems in discrete time [14]

$$
\left|\sigma_{i}[k T]\right|<\left|\sigma_{i}[(k-1) T]\right|, \quad \forall i=\overline{1,3} .
$$

The digiti red signals are low-pass filtered using a fourth-order digital butterworth filter (with a passband of $\omega_{c}=100.0$ rads/s) to remove measurement noise [13]. The flow tracking errors $e_{i}[i T]$ are then computed and combined to form the sliding surfaces (21) in discrete form

$$
\begin{gathered}
\sigma_{i}\left[k T=c_{1} e_{i}[k T]+c_{2} e_{i}[(k-1) T]+c_{3} e_{i}[(k-2) T],\right. \\
\forall i=\overline{1,3}
\end{gathered}
$$




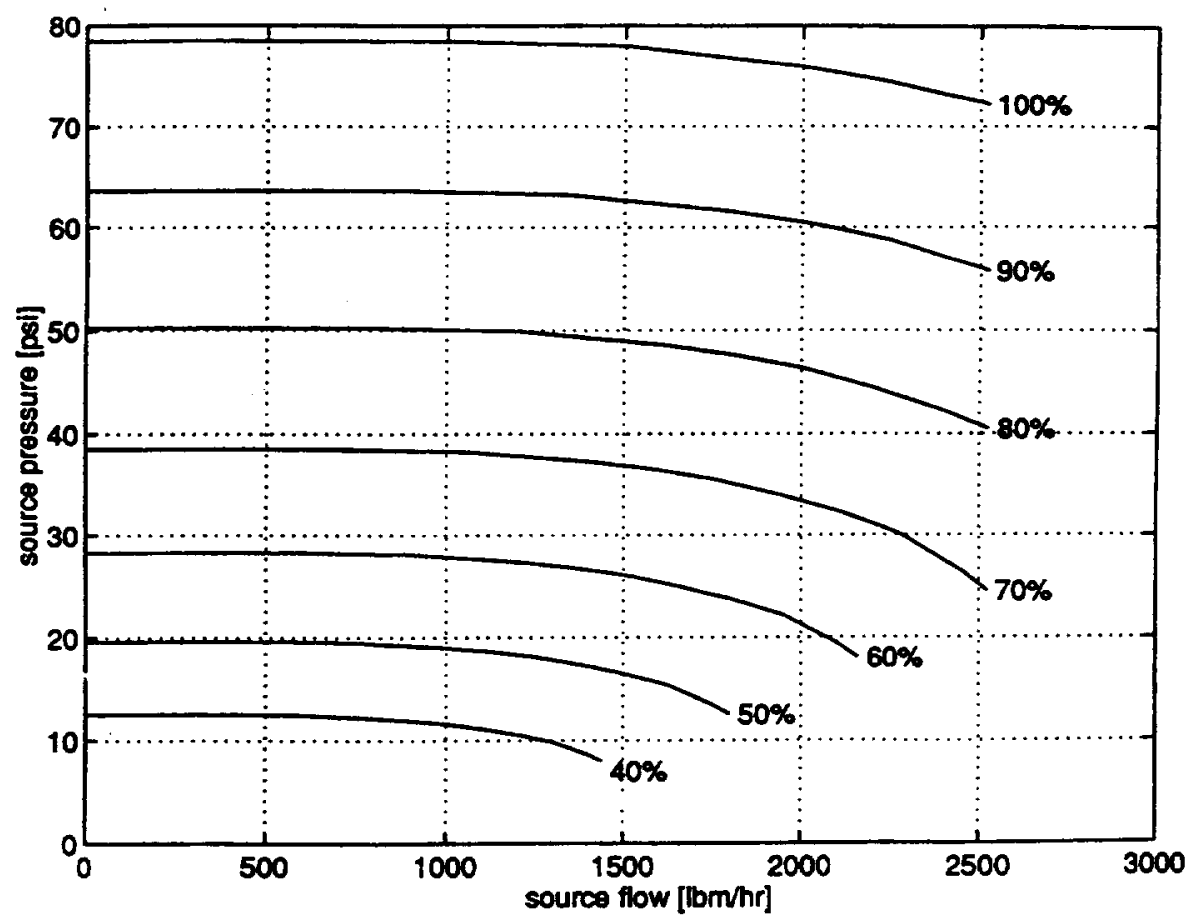

Fig. 11. Source pump map.

where $k=0,1,2, \cdots$, and

$$
\begin{aligned}
& c_{1}=\frac{225.0 T^{2}+21.0 T+1.0}{T^{2}} \\
& c_{2}=-\frac{21.0 T+2.0}{T} \\
& c_{3}=\frac{1}{T} .
\end{aligned}
$$

In general, sliding performance (specifically bandwidth) can be limited according to the discrete sample time and any unmodeled delays in the system [7]. This is shown as

$$
\omega_{n} \leq \min \left[\frac{2 \pi}{5 T}, \frac{2 \pi}{3 T_{A}}\right]
$$

where $\omega_{n}$ is the sliding surface bandwidth, $\mathrm{T}$ is the discrete sample period, and $T_{A}$ is the largest unmodeled delay. For the TCS model, the two largest unmodeled delays are through the actuator $\left(T_{A_{1}} \leq \tau\right)$ and the noise filter $\left(T_{A_{2}}=2 \pi / \omega_{c}\right)$. The delay $T_{A_{2}}$ through the noise filter is calculated as the dominate delay. Solving inequality $(41)$, it is shown that the prescribed $\therefore \quad \therefore$ e bandwidth $\left(\omega_{n}=15\right)$ specified in (37) is not unuted and is thereby realizable.

key issue in the realization of sliding mode is tt ...... of nonids. high speed switching. Defining the "smoothing" sliding mode controller

$u_{i}(k T)$

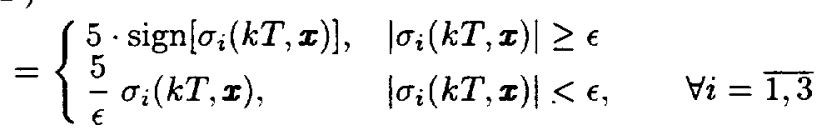

smooths the discontinuous control function (35) within $\epsilon$ vicinity of the sliding surface, thereby eliminating chattering. The boundary width $\epsilon=1.5$ is chosen to minimize steady-state error (integral control may be added if this is significant) and to achieve a tradeoff between system robustness and chattering. This tradeoff occurs because ideal nonlinear sliding control is robust (or invariant) to disturbances and uncertainties, whereas the addition of a linear region (smoothing effect) causes the system to lose some invariance to disturbances and uncertainties within the specified boundary width.

\section{Simulations}

The mathematical parameters for the numerical simulation of the TCS model are defined in the Appendix. The Runge-Kutta fourth-order algorithm with a 0.001 -s step size is used for the integration of the plant differential equations, and the discrete sliding mode controllers are scheduled every $0.005 \mathrm{~s}$. The performance of the sliding mode controllers is assessed under three operating conditions: 1) simultaneous flow profile changes in each of the three parallel paths; 2) external disturbance torques applied to the flow actuators; and 3) parameter uncertainties occurring in the model. All measurements include uniformly distributed white noise of amplitude 0 to $1 \mathrm{lbm} / \mathrm{hr}$.

Typical flow tracking profiles for paths 1,2 , and 3 are defined in Fig. 3. In response to these profiles, each path's sliding mode controller modulates its flow control valve to track the profiles. Parallel valve modulations cause the system pressure to deviate from its set-point $P^{*}$, therefore causing interaction disturbances to each path. The pressure control (a decoupling effect) is considered poorly designed or operat- 
ing in a degraded mode to intensify the interaction disturbances.

External disturbances such as unwanted torques to the control actuators originate from a variety of sources such as power delivery spikes, electromagnetic interference, and flow turbulence variations. Such torques are unmeasured and can greatly affect the performance of the TCS. For simulation purposes, torques are normalized (see Fig. 4) and added to the control input signal to represent an external disturbance.

Plant uncertainties are considered because of the difficulty in accurately modeling the system under investigation. Uncertainties can be the result of imprecise measurement instruments, improper assumptions, incorrect measurement procedures, or other measuring constraints. For this simulation, the source resistance $R_{S}$, the path flow inertance $L_{P_{1}}$, and the valve gain $k_{v_{2}}$ are considered 120,80 , and $80 \%$, respectively, off their nominal values to represent possible plant uncertainties.

The resulting flow-tracking responses in paths 1,2 , and 3 are shown in Figs. 5-7, respectively. The solid and dashed lines represent the responses to the sliding mode and PID controllers, respectively, and the dotted lines represent the setpoint profiles. Seen in Fig. 8, the system pressure deviates from its set-point significantly for both the sliding mode and PID simulations. This deviation can be a disastrous effect to controlling flow in the parallel paths. Nevertheless, the sliding mode response tracks the desired profile almost exactly, whereas the PID response deviates from the desired profile by as much a $150 \%$. This loss of performance would result in the SSFF having to shut-down; however, implementation of sliding controllers would increase system reliability and maintain system performance.

The sliding control input $\left(u_{1}\right)$ shown in Fig. 9 is smooth (does not exhibit chattering). The sliding motion $\left(\sigma_{1}\right)$ shown in Fig. 10 is within the $\epsilon$-vicinity of the sliding surface except for a few short intervals, where lack of control resources causes sliding mode to be destroyed. In summary, robust precise decoupled tracking performance is demonstrated throughout the operating range of the TCS, even in the event of system pressure control failures.

\section{CONCLUSIONS}

Sliding mode control theory has been employed in the context of the TCS problem. The discrete realization of the controllers has been developed. The most significant conclusion from this work is the determination that sliding mode control provides precise, robust, and decoupled control of flow profile tracking in the presence of interaction and external disturbances, parameter variations, and system nonlinearities. Sliding mode provides this control over the full operating range of the TCS; moreover, this control is maintain when the system pressure control is operating in a degraded mode. Excellent simulation performance and robustness is demonstrated for the discrete sliding mode controllers. Implementation of the sliding mode controllers will enhance the SSFF system's reliability and performance without additional cost or resource impacts to the system.

\section{APPENDIX \\ Mathematical Model Parameters}

Hydrau ic Submodel Data:

Path $R_{\mathrm{t}}$ sistance, $R_{P_{\mathrm{i}}}=3.57 \times 10^{-5} W_{i}\left[\mathrm{lb}_{f} \mathrm{hr}^{2}\right]\left[\mathrm{lb}_{m}\right.$ in $\left.^{2}\right\}^{-1}$

Path Inertance, $L_{P_{i}}=7.03 \times 10^{-4}\left[\mathrm{hr} \cdot \mathrm{s} \cdot \mathrm{lb}_{f}\right]\left[\mathrm{lb}_{m} \mathrm{in}^{2}\right]^{-1}$. Valve Loss Coefficients:

$\alpha=0.129\left[\mathrm{lb}_{f} \mathrm{hr}^{2}\right]\left[\mathrm{lb}_{m}^{2} \mathrm{in}^{2}\right]^{-1}$

$\beta=0.169 \mathrm{deg}^{-1}$.

Source Resistance, $R_{S}=1.0 \times 10^{-5}\left[\mathrm{lb}_{f} \mathrm{hr}^{2}\right]\left[\mathrm{lb}_{m} \mathrm{in}^{2}\right]^{-1}$. Source Inertance, $L_{S}=1.1 \times 10^{-3}\left[\mathrm{hr} \cdot \mathrm{s} \cdot \mathrm{lb}_{f}\right]\left[\mathrm{lb}_{m} \text { in }^{2}\right]^{-1}$. System Compliance, $C=0.1\left[\mathrm{lb}_{f} \mathrm{hr}\right]\left[\mathrm{lb}_{\mathrm{m}} \text { in }^{2} \mathrm{~s}\right]^{-1}$.

Source Pressure, $P_{S}=f\left(\omega_{p}, W_{S}\right)$ See Fig. 11 for map.

Actiator Submodel Data:

Valve Time Lag, $\tau=0.01 \mathrm{~s}$.

Valve Constant, $k_{v}=1.0[\mathrm{deg}][\mathrm{s} \cdot \mathrm{v}]^{-1}$.

Pump Submodel Data:

Rotor pius Load Inertia, $j_{m l}=3.1 \times 10^{-5}$ in $\cdot \mathrm{lb}_{f} \mathrm{~s}^{2}$.

Motor Gain, $k_{t}=0.33\left[\mathrm{in} \cdot \mathrm{lb}_{f}\right][\mathrm{amp}]^{-1}$.

Motor Inductance, $L_{M}=0.0012$ henrys.

Motor Resistance, $R_{M}=0.618 \Omega$.

Speed tu Torque Gain, $k_{1}=5.07 \times 10^{-7}\left[\mathrm{lb}_{f} \mathrm{~s}^{2}\right.$ in $]\left[\mathrm{rad}^{2}\right]^{-1}$.

System Pressure Set-point, $P^{*}=10.0$ psi.

Pump Speed Controller Feedforward Gains:

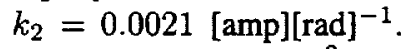

$k_{3}=0.021\left[\mathrm{amp} \cdot \mathrm{in}^{2}\right]\left[\mathrm{lb}_{f} \mathrm{~s}\right]^{-1}$.

Pump Speed Lag, $\tau_{f}=0.008 \mathrm{~s}$.

Motor Fiack EMF Gain, $k_{e}=0.042[\mathrm{v}][\mathrm{rad} \cdot \mathrm{s}]^{-1}$.

System Pressure and Pump Speed Controller Matrices:

$$
\begin{aligned}
& Q=0.0213 \quad 400.0] \quad M=\left[\begin{array}{cc}
0 & 0 \\
4.3 \times 10^{-4} & 0
\end{array}\right] \\
& \boldsymbol{B}=\left[\begin{array}{ccc}
0 & -0.1 & -0.1 \\
-4.3 \times 10^{-4} & -4.3 \times 10^{-5} & 4.3 \times 10^{-5}
\end{array}\right] \text {. }
\end{aligned}
$$

\section{ACKNOWLEDGMENT}

The authors would like to thank S. Ryan, J. Farmer, P. Vallely, an $1 \mathrm{H}$. Scofield of the National Aeronautics and Space Administrition (NASA) for their continued support.

\section{REFERENCES}

[1] U. Itki;, Control Systems of Variable Structure. New York: Wiley, 1977.

[2] V. I. I Itkin, "Variable structure systems with sliding modes," IEEE Trans. tutomat. Contr., vol. AC-22, Apr. 1977.

[3] R. A. JeCarlo, S. H. Zak, and G. P. Matthews, "Variable structure control of nonlinear multivariable systems: A tutorial," Proc. IEEE, vol. 76,$19 ; 8$.

[4] K. Og ta, System Dynamics. Englewood Cliffs, NJ: Prentice-Hall, 1978.

[5] M. E. Jackson, "Preliminary control system design and analysis for the space station furnace facility thermal control system," NASA Tech. Mem. j08476, Jan. 1995.

[6] M. E. J.sckson and Y. B. Shtessel, "Decoupled thermal control for space station furnace facility using sliding mode techniques," in Proc. Int. Forum on Space Technol. Applicat. New York: Amer. Inst. Phys., 1996.

[7] J. E. Slotine, Applied Nonlinear Control. Englewood Cliffs, NJ: Prentic:-Hall, 1991.

[8] C. D. J / hnson, "Another note on the transformation to canonical (phasevariabl :) form," IEEE Trans. Automat. Contr., vol. AC-11, Jan. 1966.

[9] V. I. U kin, Sliding Modes in Control Optimization. Berlin, Germany: Springe r-Verlag, 1992. 
[10] W. L. Brogan, Modern Control Theory. Englewood Cliffs, NJ: Prentice-Hall, 1985.

[11] M. E. Jackson, "Sliding mode thermal control system for space station furnace facility," NASA Tech. Memo. 108507, Apr. 1996.

[12] R. C. Dorf, Modern Control Systems. Reading, MA: Addison-Wesley, 1989.

[13] K. Ogata, Discrete-Time Control Systems. Englewoods Cliffs, NJ: Prentice-Hall, 1987.

[14] S. Z. Sarpturk, Z. Istefanopulos, and O. Kaynak, "On the stability of discrete-time sliding mode control systems," IEEE Trans. Automat. Contr., vol. AC-32, 1987

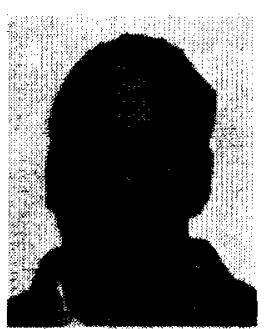

Mark E. Jackson (M'88) received the B.E.E. degree from Auburn University. Auburn, AL, in 1989 and the M.S. degree from the University of Alabama, Huntsville, in 1995.

He has been an Aerospace Engineer with the National Aeronautics and Space Administration (NASA) at the Marshall Space Flight Center since 1989 . He has worked on the control system development for the thermal and environmental control systems aboard the Space Station, and is currently working on the ascent autopilot design for the X-33 Advanced Technology Demonstrator. His research interest include dynamic system modeling, linear and nonlinear robust control, and computational analysis methods.

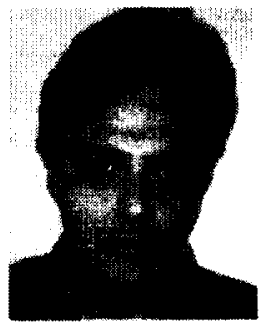

Dr. Yuri B. Shtessel (M'93) received the M.S. and $\mathrm{Ph} . \mathrm{D}$. degrees in electrical engineering with concentration in Automatic Controls from The Chelyabinsk State Technical University, Chelyabinsk, Russia in 1971 and 1978, respectively.

From 1979 to 1991 he was with the Department of Applied Mathematics and Control Science at The Chelyabinsk State Technical University. From 1991

to 1993 he was with the Electrical and Computer Engineering Department and the Department of Mathematics at The University of South Carolina. Columbia. Currently he is on faculty at the Electrical and Computer Engineering Department, the University of Alabama, Huntsville. He conducts research in sliding mode control and multiple criteria optimal control with applications to aircraft flight control systems, reusable launch vehicle control, and autonomous conventional and nuclear reactor systems of electric supply.

Since 1997 Dr. Shtessel has been a Chairman of the IEEE Control Society, Huntsville branch. 
